\section{S101 INTENSIVE CARE UNIT ADMISSION SHOULD PLAY A ROLE IN THE MANAGEMENT OF SELECTED PATIENTS WITH INTERSTITIAL LUNG DISEASE}

doi:10.1136/thoraxjnl-2012-202678.106

MC Coleman, AJ Bastin, EA Renzoni, AU Wells, TM Maher. Royal Brompton Hospital, London, UK

Introduction and Objectives Little is known about outcomes of patients with interstitial lung disease (ILD) requiring emergency admission to the intensive care unit (ICU). Historically, based on data for patients with idiopathic pulmonary fibrosis (IPF), in whom outcome is poor, there has been a reluctance to provide advanced support for individuals with ILD. The aim of this study was to evaluate outcomes of patients with ILD admitted to ICU.

Methods A retrospective review of electronic patient records, ICU database and clinical notes was undertaken for all patients with confirmed ILD admitted to ICU between January 2006 and June 2012 in a single tertiary ILD referral centre. Patients admitted electively for bronchoscopy in a high-dependency environment $(n=10$, no complications) were not included.

Results We identified 35 patients (21 male), mean age 48 (range 18-76) years. Of these, 20 were inpatients referred by the ILD team at the same hospital, 6 were admitted following deterioration after a thoracic surgical procedure and 9 were directly transferred from other hospital ICUs.

The most common diagnoses were connective tissue disease -associated ILD (6), pulmonary alveolar proteinosis, PAP (4), sarcoidosis (4), IPF (4) and hypersensitivity pneumonitis (3).

Twenty-seven (77\%) patients received invasive mechanical ventilation, 4 of whom also required extracorporeal support for gas exchange. The majority of patients received immunosuppressive therapy with corticosteroids, and over one-third received cyclophosphamide and/or rituximab whilst in ICU.

Overall, 21 (60\%) survived to ICU discharge, only 1 of whom died before hospital discharge. Sixteen (47\%) patients survived to 6 months. All 4 patients with IPF died before hospital discharge. All patients with PAP survived to 6 months. ICU length of stay for survivors was 27 (range 1-127) days and for non-survivors was 17 (1-48) days.

Conclusions Our series suggests that aggressive supportive and disease-targeted management of selected patients with ILD is appropriate, and such patients should therefore be considered for transfer to a specialist unit.

\section{Lung infection mechanisms}

\section{S102 THE EFFECT OF INFLUENZA INFECTION ON BLEOMYCIN INDUCED PULMONARY FIBROSIS}

doi:10.1136/thoraxjnl-2012-202678.107

${ }^{1} \mathrm{~A}$ Stavrou, $' \mathrm{~L}$ Jolly, ${ }^{1} \mathrm{~A}$ Habgood, ${ }^{1} \mathrm{~A}$ John, ${ }^{2} \mathrm{~T}$ Hussell, ${ }^{3} \mathrm{~A}$ Blanchard, ${ }^{1} \mathrm{G}$ Jenkins. 'University of Nottingham, Nottingham, UK; ${ }^{2}$ Imperial College London, London, UK; ${ }^{3}$ GSK, Stevenage, UK

Introduction Transforming Growth Factor-beta (TGF $\beta$ ) promotes anti-proliferative and pro-apoptotic pathways in lung epithelial cells, both of which have been implicated in the pathogenesis of IPF. TGF $\beta$ must be activated before it can mediate these events. Acute exacerbations of IPF are characterised by widespread epithelial cell apoptosis. The precise cause of these exacerbations is not known. The Influenza A virus is a single-stranded segmented RNA virus that infects epithelial cells leading to cell death and injury, and can also activate TGF $\beta$. The role of infection in acute exacerbations of IPF is unclear. The aim of this study is to investigate the effect of influenza infection on bleomycin-induced pulmonary fibrosis and TGF $\beta$ activation in vivo.

Materials and Methods $60 \mathrm{U}$ of bleomycin was instilled into the lungs of 6-8 week old male $\mathrm{C} 57 \mathrm{Bl} / 6$ mice. After 28 days mice were exposed intranasally with 10, 20 Units of influenza virus ' $x 31$ ' or $\mathrm{PBS}$, and the lungs harvested 5 days later. Bronchoalveolar lavage (BAL) was performed and lung tissue harvested for mRNA analysis, histology and hydroxyproline levels. All animal studies were ethically reviewed and carried out in accordance with Animals (Scientific Procedures) Act 1986 and the GSK Policy on the Care, Welfare and Treatment of Animals.

Results Mice exposed to bleomycin and infected with influenza lost less weight compared with saline-exposed influenza-infected animals. However, the lungs from bleomycin-exposed, influenzainfected mice showed increased lung damage with more matrix deposition on trichrome staining than saline-exposed, influenzainfected mice. Saline-exposed, influenza-infected mice demonstrated the anticipated dose dependent increase in BAL lymphocytosis as well as apoptosis staining in histological TUNEL assessment. However, in bleomycin-exposed mice, influenza infection did not promote enhanced BAL lymphocytosis or apoptosis. However, influenza appeared to enhance the fibrotic response demonstrated by an increase in matrix deposition on masson's trichrome and increased lung hydroxyproline levels in influenza infected bleomycin exposed mice, as early as 5 days post infection.

Conclusions These data suggest that influenza infection may exacerbate lung fibrosis by promoting epithelial apoptosis.

\section{S103 ANTI-PSEUDOMONAL BACTERIOPHAGE COCKTAIL REDUCES INFLAMMATORY RESPONSES IN THE MURINE LUNG}

doi:10.1136/thoraxjn-2012-202678.108

${ }^{1} \mathrm{R}$ Pabary, ${ }^{2} \mathrm{C}$ Singh, ${ }^{3} \mathrm{~S}$ Morales, ${ }^{1} \mathrm{~A}$ Bush, ${ }^{4} \mathrm{~K}$ Alshafi, ${ }^{5} \mathrm{D}$ Bilton, ${ }^{2} \mathrm{EWFW}$ Alton ${ }^{3} \mathrm{~A}$ Smithyman, $1 \mathrm{JC}$ Davies. 'Department of Paediatric Respiratory Medicine, Royal Brompton Hospital, London; 'United Kingdom, National Heart and Lung Institute, Imperial College, London, United Kingdom; ${ }^{3}$ Special Phage Services, Brookvale, Australia; ${ }^{4}$ Department of Microbiology, Royal Brompton Hospital, London, United Kingdom; ${ }^{5}$ Adult CF Unit, Royal Brompton Hospital, London, United Kingdom

Bacteriophages are naturally occurring viruses that specifically target and infect bacteria and, unlike antibiotics, are able to multiply at infection sites and adapt to resistant bacteria. We hypothesise that bacteriophage cocktails may be useful against $P$. aeruginosa $(\mathrm{Pa})$ in cystic fibrosis and tested this in a murine infection model.

Two strains of Pa were assessed: a) a clinical strain from an adult CF patient, b) a laboratory strain. Both strains were shown to be sensitive to a novel anti-Pseudomonal bacteriophage cocktail on a standard plaque assay.

Adult BALB/c mice were inoculated intranasally with 50ul of $\mathrm{Pa}$ followed by 20ul of bacteriophage cocktail (treated, $n=21$ ) or SM buffer (control, $n=21$ ). Twelve mice were sacrificed at 24 hrs after infection and the others at $48 \mathrm{hr}$. Bronchoalveolar lavage was serially log diluted, cultured at $37^{\circ} \mathrm{C}$ and the remainder centrifuged and supernatant stored at $-80^{\circ} \mathrm{C}$ for future analysis of soluble inflammatory markers. Total cell counts were determined using a haemocytometer. Non-quantitative splenic cultures were performed.

Results All mice treated with bacteriophage $(n=6)$ had cleared infection at $24 \mathrm{hrs}$ compared with none of the controls $(n=6)$ (median [range] CFU/ml 0 [0-0] vs. 1305 [190-4700], p<0.01); inflammatory cell counts did not differ. At the $48 \mathrm{hr}$ time point most mice had cleared the infection, with no phage-related differences. However, treated mice demonstrated significantly fewer inflammatory cells in BAL compared with controls (median [range] 4.50 [2.84$5.86] \times 10^{4} / \mathrm{ml}$ vs. 9.12 [6.93-13.86], $\mathrm{p}<0.01$ for the clinical strain; median [range] 6.04 [5.56-10.60] $\times 10^{4} / \mathrm{ml}$ vs. 9.72 [8.56-15.28], $\mathrm{p}<0.01$ for the laboratory strain). Differential cell counts and measurements of soluble inflammatory mediators are underway.

$\mathrm{BALB} / \mathrm{c}$ mice successfully cle ared this dose of Pa by $48 \mathrm{hrs}$, with an accompanying acute cellular inflammatory response. The reduction in cell number following co-administration of a bacteriophage 